ARTICLE

\title{
Simulation of GEM-TPC Prototype for the Super-FRS Beam Diagnostics System at FAIR
}

\author{
Matti KALLIOKOSKI* \\ Helsinki Institute of Physics, P.O.BOX 64, FIN-00014 University of Helsinki, Finland
}

\begin{abstract}
The Superconducting Fragment Recoil Separator will be a key part of the experiments of the NuSTAR collaboration. The beam of the separator will have heavy ion projectiles that are steered to the experimental area. With the Time Projection Chambers with Gas Electron Multiplier amplification (GEM-TPC), diagnostics of the beam properties can be performed. The performance of the components of the design of a GEM-TPC prototype has been studied with Geant4 and Garfield simulation tools.
\end{abstract}

KEYWORDS: GEM-TPC, Super-FRS, FAIR, NuSTAR, Geant4, Garfield

\section{Introduction}

The Superconducting Fragment Separator (Super-FRS) will be one of the key parts of the future Facility of Antiproton and Ion Research (FAIR) that will be built in Darmstadt as an extension of the current GSI facility. ${ }^{1-2)}$ The SuperFRS will serve the needs of the experiments of Nuclear Structure, Astrophysics and Reactions (NuSTAR) collaboration. It will perform in-flight separation of all primary beams up to ${ }^{238} \mathrm{U}$ and via fission of ${ }^{238} \mathrm{U}$ beams. A diagram of the Super-FRS is seen in Fig. 1.

The experiments of the NuSTAR collaboration will use common beam diagnostics system. This system will be used for adjusting the separator, beam monitoring, particle identification, tracking and characterization of the projectiles. The beam diagnostics system consists of different types of detectors that will be placed in detector ladders of beam diagnostics stations.

Beam monitoring at slow extraction with extraction times above $100 \mathrm{~ms}$ can be done with Time Projection Chambers

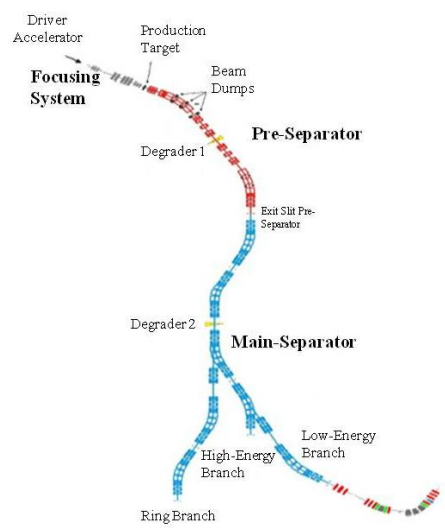

Fig. 1 Diagram of the Superconducting Fragment Separator

*Corresponding author, E-mail:K.Kalliokoski@helsinki.fi

(C) 2011 Atomic Energy Society of Japan, All Rights Reserved.

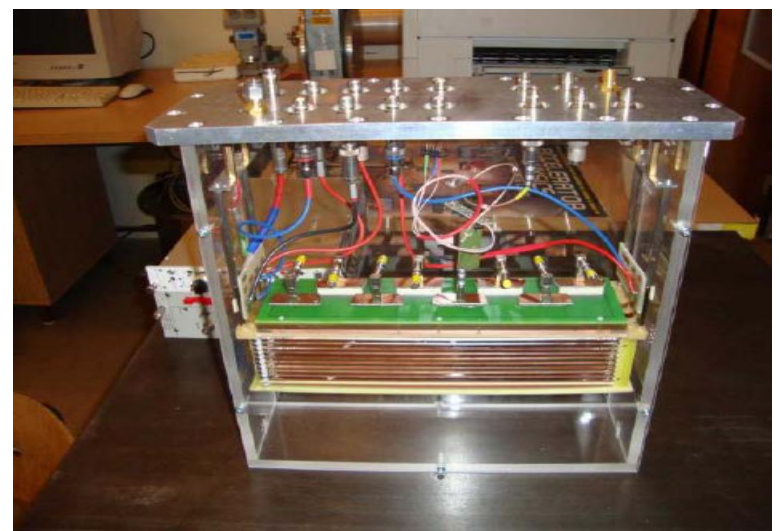

Fig. 2 GEM-TPC prototype in laboratory measurements in Bratislava

with Gas Electron Multiplier amplification at the readout (GEM-TPC). ${ }^{3)}$ Groups from Helsinki and Bratislava have designed and built a prototype GEM-TPC detector that will be used to analyze the performance of the detector type in the Super-FRS environment. ${ }^{4-5)}$ Picture of assembled GEMTPC prototype in laboratory measurements can be seen in Fig. 2.

Simulation models to analyze the performance of the GEM-TPC detectors in Super-FRS environment have been developed using Geant4 toolkit and Garfield simulation program. $^{6-7)}$

\section{Simulations}

\section{Simulation Tools}

Geant4 is a software toolkit for simulating particle interactions with matter. It is written in $\mathrm{C}++$ to fully utilize the object oriented methods. With Geant 4 classes a detector model drawn with $\mathrm{CAD}$ modeling tools can be imported into 


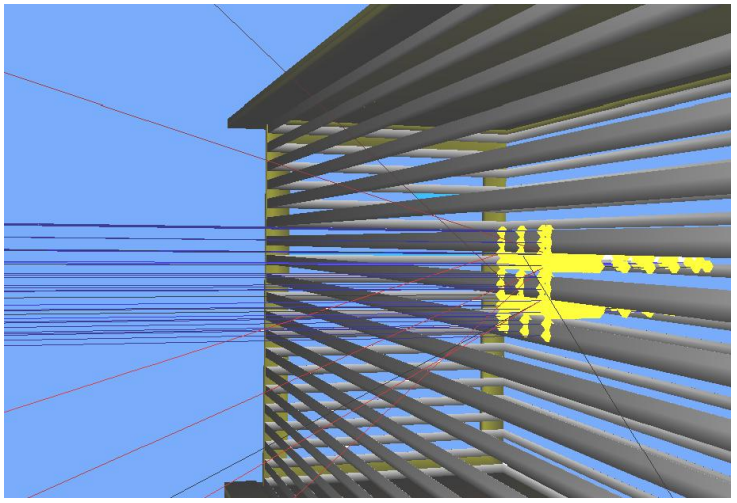

Fig. 3 Geant 4 simulation of heavy ion beam entering the drift volume of the GEM-TPC detector

Geant4 and the interaction of particles with the detector components can be studied.

To import the CAD models to Geant4 they are first converted into Geometry Description Markup Language (GDML) format. ${ }^{8}$ The GDML is application independent format based on XML that can be used to interchange geometries between different applications. In Geant4 the detector construction from GDML geometry is done with the GDMLProcessor which parses a geometry file and returns the pointer to world volume.

Geant 4 can describe the particle paths and the secondary particles that have been generated in the interactions but it does not have description for drift and effects of the electric and magnetic fields inside the gas detectors. Therefore, for these parts Garfield simulation program has been used.

Garfield is a FORTRAN based program built to simulate drift chambers. It has an interface to Magboltz and Heed simulation tools for calculating the properties of the electron transportation and ionization of gas molecules by particles traversing the gas volume. ${ }^{9-10)}$ Garfield accepts field maps computed by finite element (FEM) software as basis of its calculations but with the recent inclusion of interface to the nearly exact Boundary Element Method (neBEM) the field maps can be created stand-alone. ${ }^{11)}$ In the simulations Garfield version 7.36 was used.

\section{Drift Volume}

The drift volume of the Time Projection Chamber model in the simulations had the dimensions of $40 \mathrm{~cm}$ in width, $20 \mathrm{~cm}$ in height and the thickness of the volume was $5 \mathrm{~cm}$. The walls of the TPC, or the field cage, consisted of aluminum coated Mylar strips with strip width of $2 \mathrm{~mm}$ and pitch of $1 \mathrm{~mm}$. Ar- $\mathrm{CO}_{2}$ (70:30) gas composition was used in the simulations. In Fig. 3, Geant4 simulation of projectiles entering the detector volume releasing secondary electrons from the drift volume can be seen.

Different beam spot sizes were set using the PrimaryGeneratorAction class of Geant4. The distribution of the projectiles was set to be Gaussian. In Fig. 4, distribution of secondary electrons from $100 \mathrm{MeV}$ beam with $\sigma_{\mathrm{x}}=1 \mathrm{~mm}$ and $\sigma_{\mathrm{x}}=4 \mathrm{~mm}$ projectiles of ${ }^{134} \mathrm{Sb},{ }^{132} \mathrm{Sn}$ and ${ }^{130} \mathrm{In}$ can be seen.
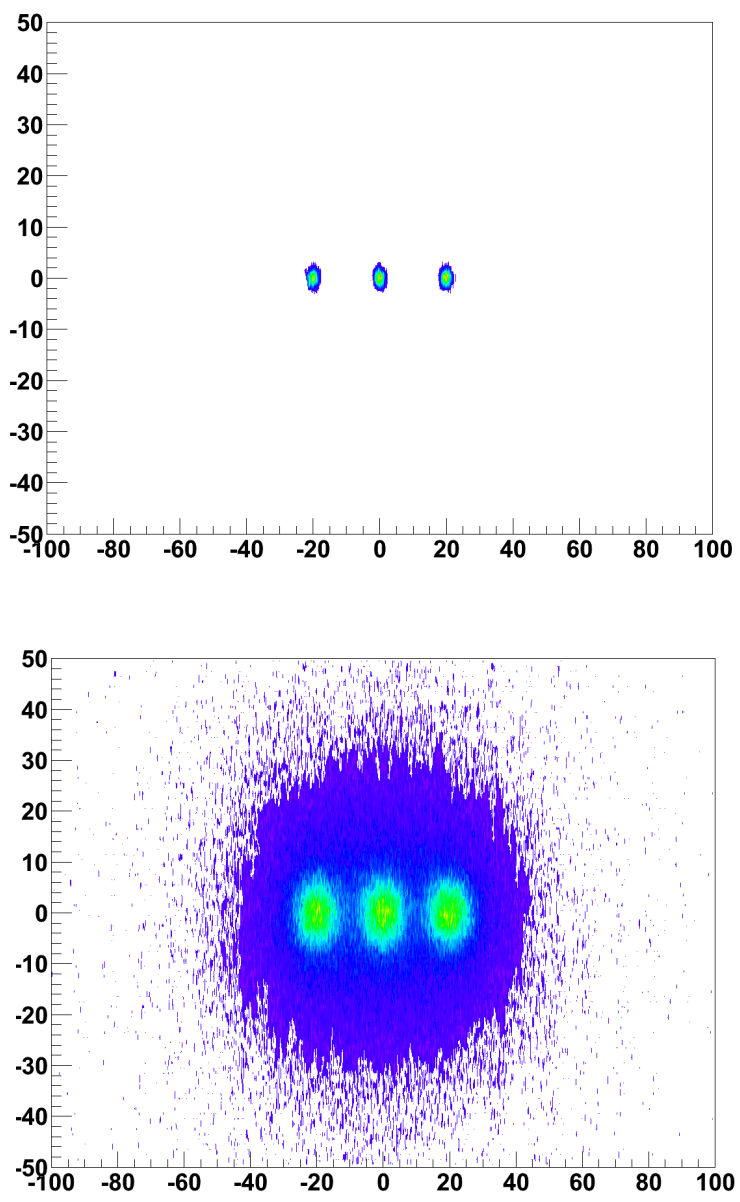

Fig. 4 Simulated distribution of secondary electrons from beam of $\sigma_{x}=1 \mathrm{~mm}$ (top) and $\sigma_{x}=4 \mathrm{~mm}$ (bottom) projectiles of ${ }^{134} \mathrm{Sb}$, ${ }^{132} \mathrm{Sn}$ and ${ }^{130}$ In with beam energy of $100 \mathrm{MeV}$

Geometries constructed for and in Geant 4 cannot be imported directly to Garfield for field calculations. Therefore, the detector model had to be reconstructed by the tools within the Garfield program. In Garfield version 7.36, limitations in optimization of the geometries in the neBEM solver required reduction of the number of strips electrodes used in the walls of the field cage. Thus, the height of the sensitive volume in the Garfield simulations was $120 \mathrm{~mm}$. For the studies of secondary electron drift from the beam projectiles the distance is sufficient.

The field cage was constructed within \&CELL section of the Garfield program using the SOLIDS to build the conductive electrode strips that form the field cage. The voltage on the strips was stepped down along the height of the drift to evoke a drift field of $250 \mathrm{~V} / \mathrm{cm}$. To the top of the volume a conductive drift foil in the same potential as the topmost strip was added. Conductive plate was also added to the bottom of the drift volume in the potential of top of the first GEM-foil. The quality of the field mapping from the neBEM solver was compared with studies made with commercial FEM software.

In \&GAS section, Garfield calls Magboltz and Heed simulation tools for the calculations of the properties of the 

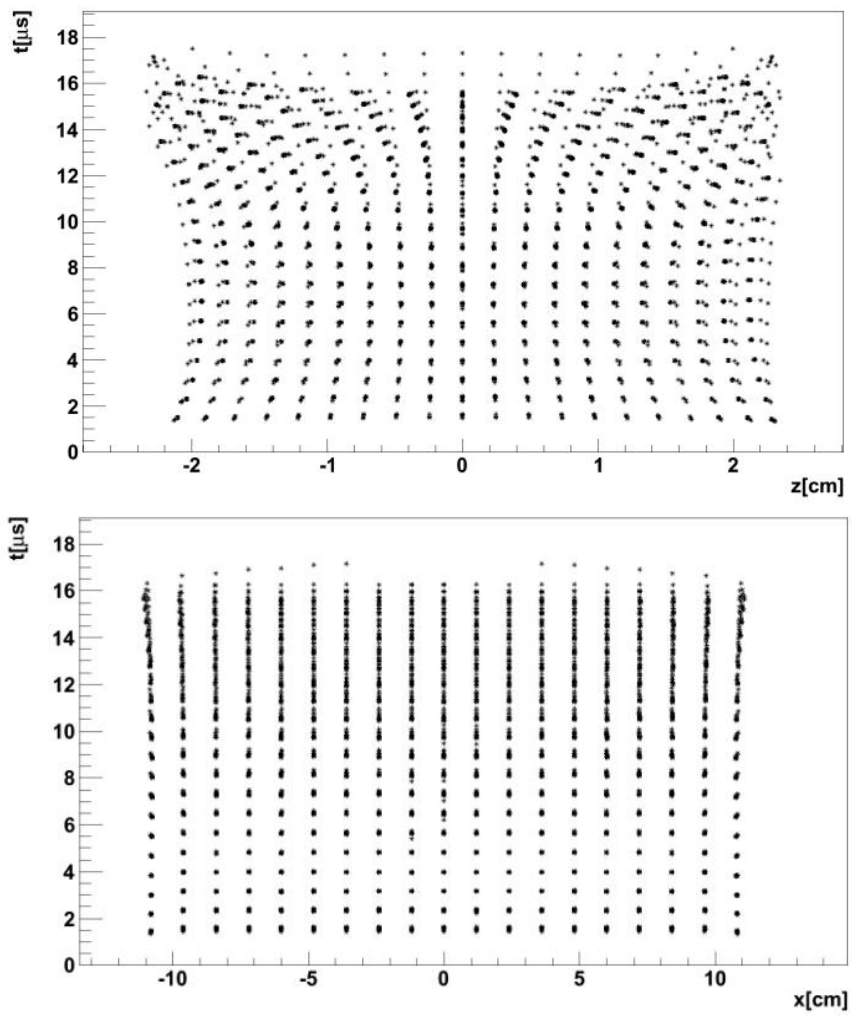

Fig. 5 Drift times projected to depth and width of the drift volume. The figure on top shows the drift times in comparison with the depth and the bottom one with the width of the volume.

selected gas mixture. In the simulations $\mathrm{Ar} / \mathrm{CO}_{2}$ (70:30) mixture was studied at room temperature and standard pressure.

The actual drift simulations are done in \&DRIFT section. In this section the secondary electrons that were generated in the interactions with the beam projectiles are drifted towards the GEM amplification part of the detector. For the field drift volume, the Runge-Kutta-Fehlberg method in integration was used to calculate the paths of the drifting electrons. For this part the method was used to reduce the computation time and the requirements for the CPU.

In Fig. 5, the drift times from different parts of the drift volume are presented. In optimal case the drift times would be linear with no overlapping. Since the drift volume depth is small compared to the height of the detector some overlapping in the drift times can be seen. The top figure is the view from the end of the detector and the bottom is from the front side. In the plots, the full width of the detector is not shown since the results from the Geant 4 are focused on the area shown.

The pileup in drift times at the top of the drift volume is caused by the depth of the field cage. The paths of the drifting electrons are curved and longer closer to the front and back of the detector walls. Figure $\mathbf{6}$ shows the mean values of the drift times over the drift distance.

Deviation of drifting electrons from initial position over a drift distance of $100 \mathrm{~mm}$ can be seen in Fig. 7. The Xprojection of the beam at the top of the GEM-amplification can be obtained directly from the Geant 4 simulations since

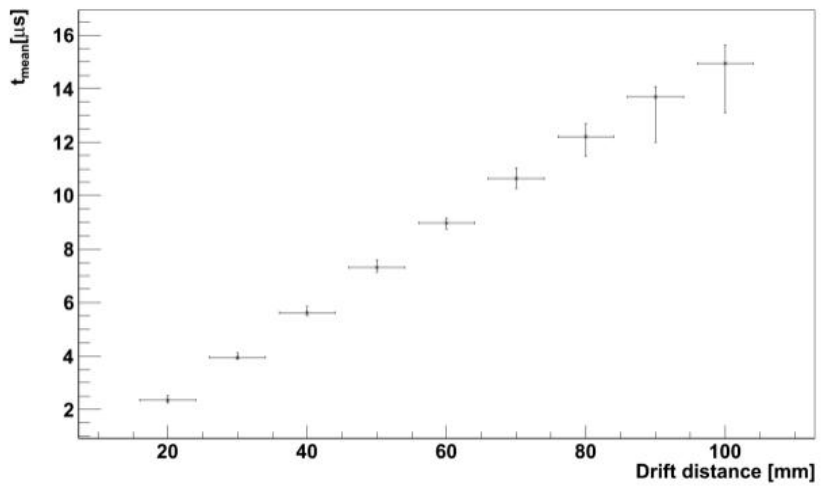

Fig. 6 Mean drift time over different drift distances

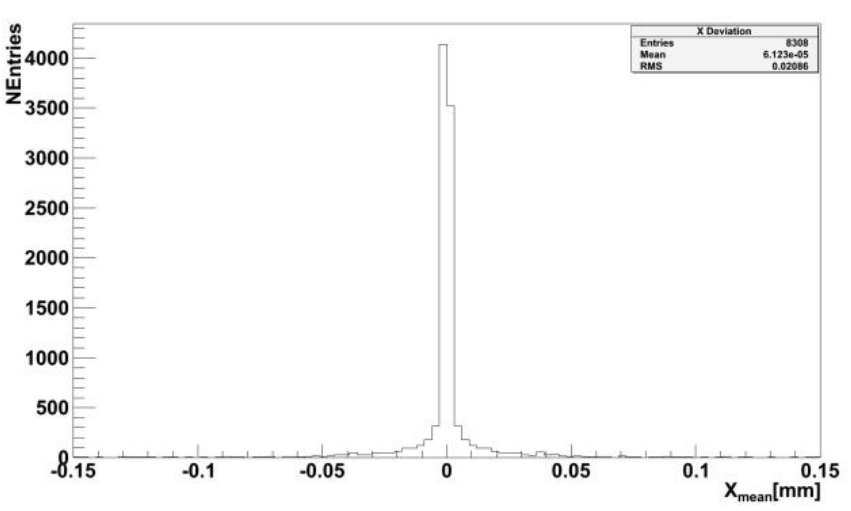

Fig. 7 Distribution of electrons after drift over a distance of $100 \mathrm{~mm}$ in $\mathrm{Ar} / \mathrm{CO}_{2}$ at drift field of $250 \mathrm{~V} / \mathrm{cm}$

there are no deviations in the drift. The pileup in Z-direction will affect only to the length of the signal.

\section{GEM Amplification}

Standard GEM consists of $50 \mu \mathrm{m}$ thick Kapton foil that has $5 \mu \mathrm{m}$ copper coating on both sides. The whole plane is perforated with a large number of bi-conical holes with external diameter of $70 \mu \mathrm{m}$, internal diameter of $50 \mu \mathrm{m}$ and pitch of $140 \mu \mathrm{m}$ on staggered rows. When a potential difference is set between the metal electrodes, a high electric field is generated inside the holes.

The modeling of a GEM-hole was done within \&CELL section of Garfield utilizing again the neBEM interface for field mapping. A hole in the middle of a GEM-foil has five neighbors. With SOLIDS commands a cell with a central hole and its neighboring holes with the neighboring holes of the neighbors was constructed. Thus the studied area had total of 19 holes. This was done to have an accurate field on top and bottom of the central hole that was studied. Figure 8 shows a cutout view of an area around the central hole showing the five neighboring holes.

Central hole was set to have the highest resolution while the surrounding holes were left to be optimized by the automatic routines. To study the drift and induction fields on top and on the bottom of the foil, conductive planes were added $2 \mathrm{~mm}$ above and below the foil. 


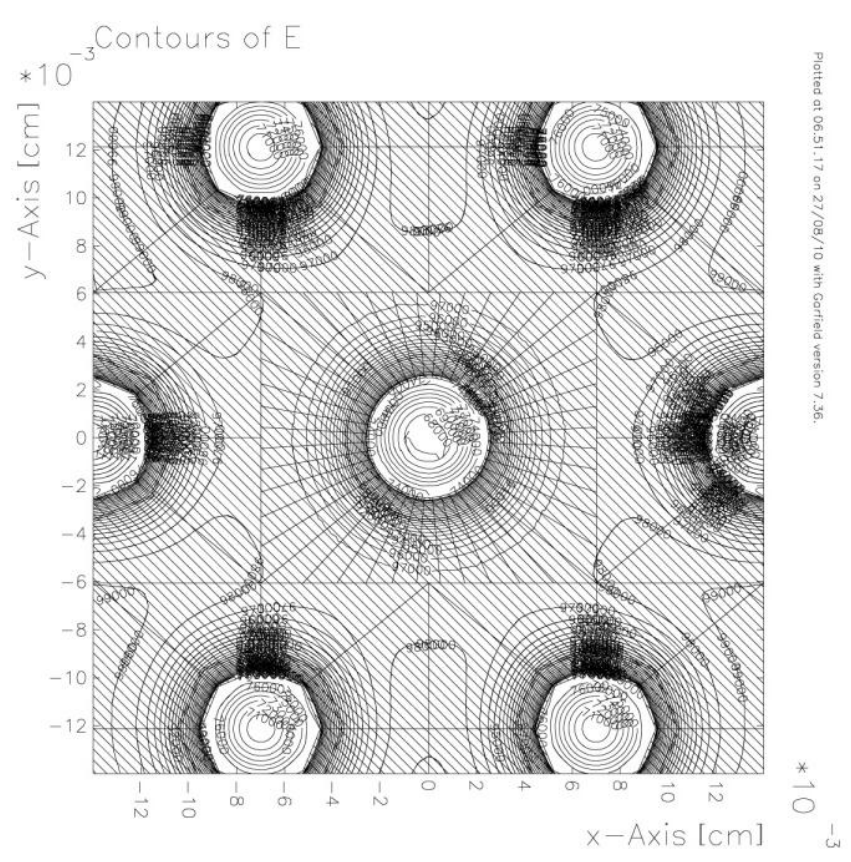

Fig. 8 Cutout of the GEM-foil cell used in the studies

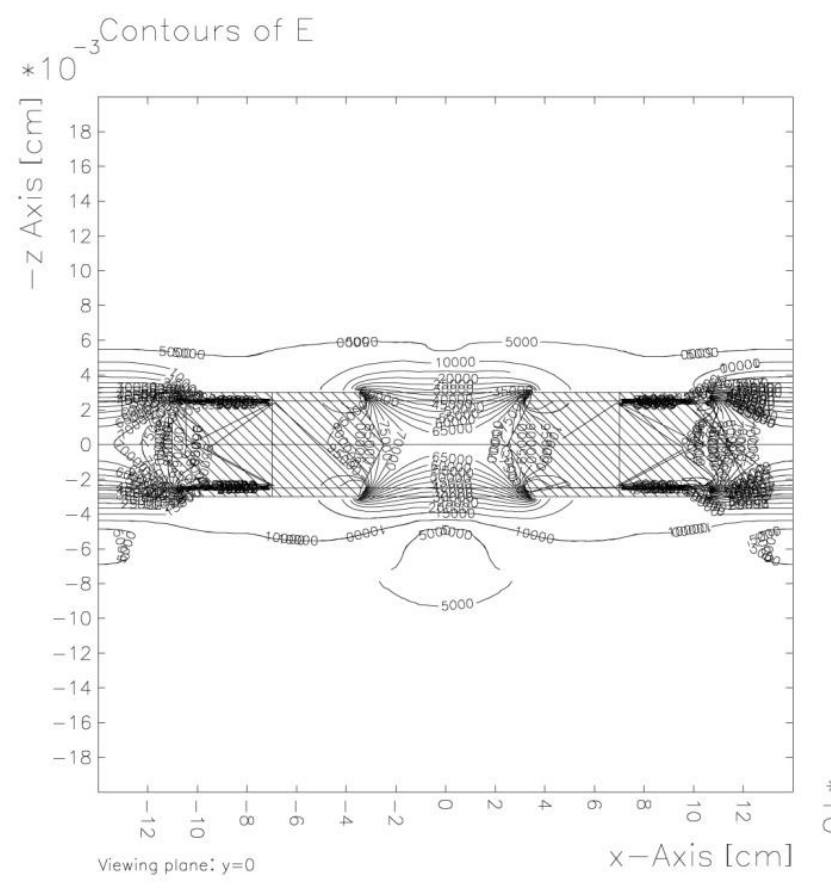

Fig. 9 Equipotential lines of a GEM-foil with drift field of $250 \mathrm{~V} / \mathrm{cm}$ and induction field of $3 \mathrm{kV} / \mathrm{cm}$. The amplification voltage is $400 \mathrm{~V}$.

The drift field on top of the first foil was set to same value as inside the field cage $250 \mathrm{~V} / \mathrm{cm}$. The induction field at the bottom was set to $3 \mathrm{kV} / \mathrm{cm}$. The amplification voltages of $400 \mathrm{~V}, 350 \mathrm{~V}$ and $300 \mathrm{~V}$ were studied. The field lines on top and bottom of the first configuration with amplification voltage of $400 \mathrm{~V}$ is plotted in Fig. 9.

The avalanche inside a hole in the GEM-foil was studied with the microscopic avalanche method of \&DRIFT section. This method tracks the electron path in molecular level using Monte Carlo techniques. The tracking method classifies

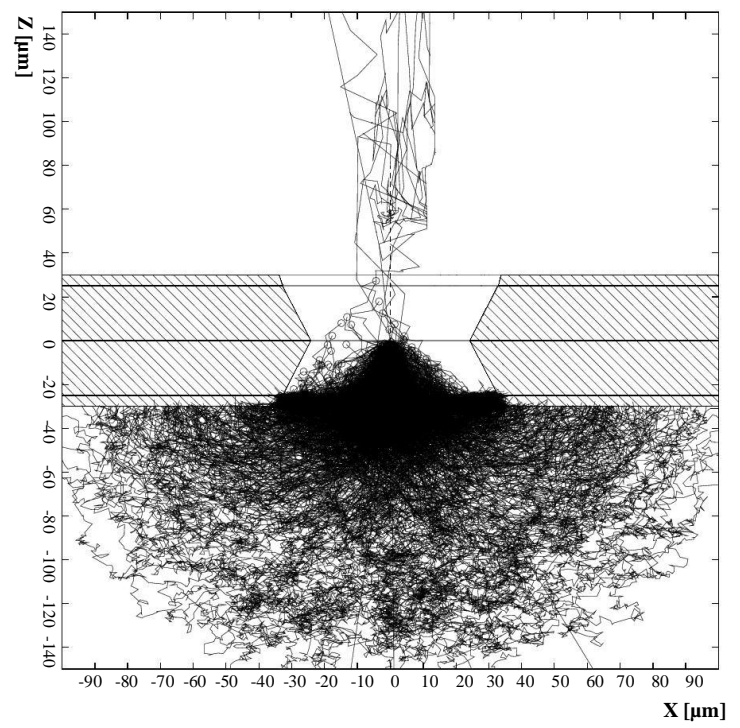

Fig. 10 Avalanche from electrons drifting to a GEM-hole. The detector gas is $\mathrm{Ar} / \mathrm{CO}_{2}$ (70:30) in standard pressure and in room temperature.

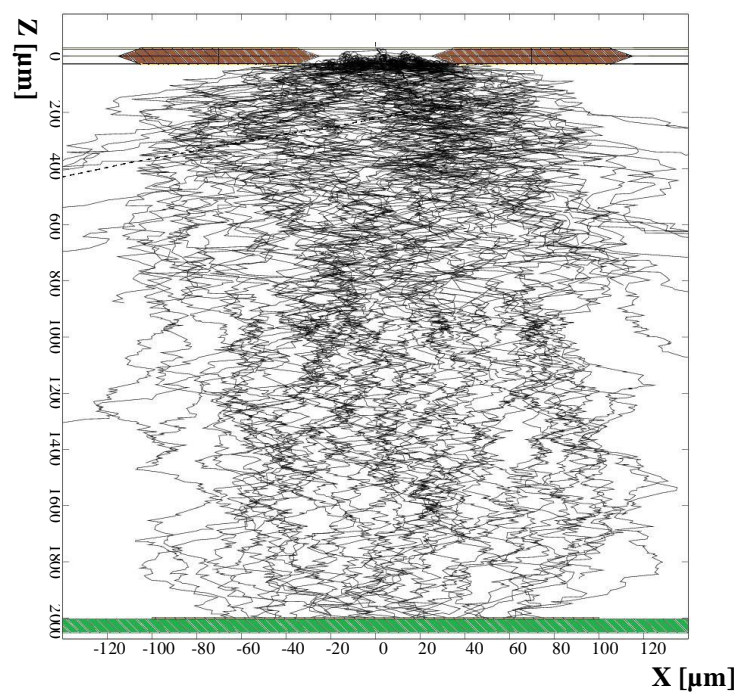

Fig. 11 Garfield simulation of electrons drifting from a GEMhole to the readout board over induction gap of $2 \mathrm{~mm}$ in $\mathrm{Ar} / \mathrm{CO}_{2}$ (70:30). Induction field is $3 \mathrm{kV} / \mathrm{cm}$ and the amplification voltage is $400 \mathrm{~V}$.

the collisions along the path as elastic, inelastic, superelastic, attachment or ionization from the results given by Magboltz. Magboltz contains for each gas composite separate cross section for each type of interaction. In Fig. 10, an avalanche created by electrons drifting to a GEM-hole is plotted.

\section{Readout}

The readout board has strips with strip width of $200 \mu \mathrm{m}$ and pitch of $500 \mu \mathrm{m}$. The strips are divided in two parts along the width of the detector giving two strip lengths that slightly differ from each other. The gap between the strips at center of the board is $500 \mu \mathrm{m}$. 
The number of secondary electrons coming from the drift volume is large and thus no large amplification is required. The width of the charge cluster from single electron can be adjusted to be narrow enough to have interference only with one strip and its neighbors. Figure 11 shows the electrons created in an avalanche in GEM-hole drifting towards a readout strip. Main part of the charge is collected by one strip of the readout board. The induction field is $3 \mathrm{kV} / \mathrm{cm}$ drift and the amplifying voltage over the GEM is $400 \mathrm{~V}$.

\section{Conclusions}

A model to study performance of a GEM-TPC detector at the Super-FRS environment has been build. Geant 4 was used to study the interaction of the beam projectiles with the sensitive volume of the detector. By combining the results with drift field studies done with Garfield, a full analysis of the detector efficiency can be done.

For both drift and GEM-amplification studies, interface to the neBEM solver has been used for the field mapping of the electric field inside the detector. Though with the interface only elementary shapes can be studied, from the simulations it can be seen that these already allow full studies of a complex detector system.

From the results it can be seen that a GEM-TPC detector can operate in Super-FRS environment as beam monitor in slow extraction. As seen in Fig. 7, the accuracy along the width of the detector gives a good position resolution for the beam correction and adjustment.

The GEM-amplification stage can be used to adjust the cluster shapes to be optimal for the readout system. Since the amount of secondary electrons generated in the drift volume is large amplification voltages of the foils can be kept low.

Prototype GEM-TPC detectors have been constructed and will be studied with test beams of FRS-separator at GSI. The results of the measurements will be used to benchmark the simulation results. The measurement results will be used together with the simulations in the design process of the final version of the GEM-TPC detectors that will be used in the Super-FRS.

\section{References}

1) M. Winkler et al., "The status of the super-FRS in-flight facility at FAIR," Nucl. Instr. Meth. Phys. Res., B266, 4183-4187 (2008).

2) G. Rosner, "Future facility: FAIR at GSI," Nucl. Phys. B Proc. Suppl., 167, 77-81 (2007).

3) M. Killenberg et al., "Modelling and measurement of charge transfer in multiple GEM structures," Nucl. Instr. Meth. Phys. Res., A498, 369-383 (2003).

4) F. Garcia et al., "GEM-TPC prototype for beam diagnostics of Super-FRS in NUSTAR experiment - FAIR," 2009 IEEE NSS Conference Record, Oct. 25-31, Orlando, USA, N13-7 (2009), [CD-ROM].

5) M. Kalliokoski et al., "GEM-TPC trackers for the Super-FRS at FAIR," Proceedings of IPAC'10, May 23-28, Kyoto, Japan, paper MOPD082 (2010).

6) S. Agostinelli et al., "Geant4 - a simulation toolkit," Nucl. Instr. Meth. Phys. Res., A506, 250-303 (2003).

7) R. Veenhof, "Garfield, recent developments," Nucl. Instr. Meth. Phys. Res., A419, 726-730 (1998).

8) R. Chytracek et al., "Geometry description markup language for physics simulation and analysis applications," IEEE Trans. Nucl. Sci., 53[5], Part 2 (2006).

9) S. F. Biagi, "A description of the Magboltz program with results compared to experiment," Nucl. Instr. Meth. Phys. Res., A421, 234-240 (1999).

10) I. B. Smirnov, "Modeling of ionization produced by fast charged particles in gases," Nucl. Instr. Meth. Phys. Res., A554, 474-493 (2005).

11) S. Mukhopadhyay, N. Majumdar, "A study of threedimensional edge and corner problems using the neBEM solver," Eng. Anal. Bound. Elem., 33[2], 105-119 (2009). 